\title{
Los Orígenes Estadisticos de las Encuestas de Opinión
}

\author{
Beatriz Mañas Ramfrez \\ U.N.E.D. \\ bmanas@bec.uned.es
}

Recepción: diciembre 2004

Aceptación: febrero 2005

\section{INTRODUCCIÓN}

Cuando se trata de investigar los dispositivos de «medición» de determinada entidad, nos enfrentamos inmediatamente a una serie de cuestiones que se relacionan, por una parte, con la realidad y la naturaleza del objeto a medir, y por otra, con el proceso de medición mismo, es decir, con la pertinencia de la herramienta utilizada y la calidad del resultado de dicho proceso, en términos de su «validez» y «fiabilidad». En realidad, ambas cuestiones se encuentran en permanente tensión debido a los problemas que plantea conocer la calidad de una «medición» cuando, al tiempo, se debate si el objeto es o no real, o simplemente producto de una convención.

Esta problemática, largamente inscrita en el debate de las ciencias sociales, está presente de modo muy particular en el caso de la investigación de la «opinión pública» porque, ¿hasta qué punto se puede establecer una medida numérica para dar cuenta de una entidad que, en el caso de que exista, es tan difícilmente objetivable y cuantificable?

Señalar desde el comienzo que la «opinión pública» es un concepto problemático, complejo, que además nunca ha tenido un significado unívoco y universal para todo tiempo y lugar, es algo que no resultará, desde luego, sorprendente. Se trata de un concepto que se ha ido modificando paulatinamente como resultado de circunstancias histórico-sociales cambiantes y que, además, se ha visto afectado en su misma definición por la metodología utilizada, por el dispositivo que en cada momento ha servido para objetivarlo, para exteriorizarlo, para dar cuenta del mismo. En el presente artículo trataremos, así, de presentar, tanto los comienzos de la noción de opinión pública, como los orígenes matemáticos de la herramienta que hoy en día se utiliza de forma predominante para estudiar e investigar dicho fenómeno: la encuesta estadística. 


\section{LOS ORÍGENES DEL CONCEPTO: OPINIÓN DEL PÚBLICO vS OPINIÓN DE LAS MASAS}

La aparición del sentido moderno de la noción de «opinión pública» como aquélla propia del «público ilustrado» que, «por medio de la discusión crítica en la publicidad, acaba por destilar la opinión verdadera» (Habermas 1994: 132), sólo es posible cuando emerge una nueva cultura política que ha surgido al hilo de la transformación de los sistemas de relaciones económicas y de la esfera familiar burguesa, y que se apoya en la constitución de una burguesía ilustrada que va a hacer uso de determinados lugares e instituciones (clubes, cafés, prensa...) como entorno propicio para que esta élite «éclairée» sea capaz de ejercer una permanente crítica del poder político a través de la información colectiva, la discusión y el uso del razonamiento. Estas circunstancias, constituirán en Francia el contexto de los debates que tienen lugar, a partir de mediados del siglo XVIII, en el momento en que la «opinión» pasa del ámbito privado al ámbito de lo «publico», cuando distintos actores sociales pretenden erigirse como sus «portadores legítimos». Es decir, se va a pasar de una concepción «negativa», según la cual la opinión pública designa el conjunto de ideas y de juicios compartidos por un grupo social asociados a la tradición, el honor, la estima, los hábitos, los prejuicios, las conjeturas, más bien circunscrita al domino de los comportamientos y las actitudes privadas, hasta una concepción moderna más «positiva» donde la crítica popular contra el gobierno real, agitada por la prensa clandestina y ligada, como se ha dicho, al desarrollo de una burguesía ilustrada, la dota de un sentido más cercano al de «control social» hacia los gobernantes.

Sin embargo, esta forma de concebir la «opinión pública» como el «resultado ilustrado de la reflexión común y pública sobre los fundamentos del orden social» (Blondiaux 1998: 130) tiene sus consecuencias en cuanto supone una forma de delimitar quién forma parte del "sujeto» de la misma. Así, nos encontramos a partir del siglo XVIII con una «opinión pública» que no engloba al «pueblo», ya que éste, por constituir una «masa ciega», «ruidosa», «ignorante», «cambiante», sólo puede tener una opinión «común», «vulgarizada», como corresponde a una multitud que no está convenientemente «informada»y, por tanto, no tiene la capacidad para saber lo que le conviene. Obviamente, la «razón» será utilizada como elemento legitimador de la capacidad para erigirse como representante de la opinión verdadera y, por tanto, es así como encontrará justificación el uso del concepto de «opinión pública» por las élites burguesas para legitimar sus reivindicaciones políticas frente a los gobernantes del Antiguo Régimen, haciéndose portadoras de una razón que, a su vez, las legitima como representantes de la voz del pueblo.

Todo ello hace que la «opinión pública» no se entienda todavía como el resultado de la adición estadística del número más alto, pues la cantidad se equipara todavía a la «muchedumbre», a la «opinión popular», sinónimo de «pasiones desordenadas e inestables». Sin embargo, llega un momento en que cabe preguntarse cómo es posible la supervivencia de un concepto que había encon- 
trado su fundamento y su razón de ser en la crítica del poder real, una vez que las élites burguesas e ilustradas logran sus objetivos tras la Revolución Francesa. Evidentemente, la única forma en la que puede sobrevivir es cambiando su fuente de legitimidad: la «opinión pública necesita traspasar su elemento legitimador desde la razón hasta la cantidad, hasta el número de personas que la suscriben. Es de esta forma como el «pueblo» se convierte en una categoría central del discurso político, y como los conceptos de «opinión pública» y de opinión «popular», «común», que hasta entonces habían estado separados, encuentran ahora su identificación. Así, el hecho de que, al menos simbólicamente, las masas hayan entrado a formar parte del sujeto de la opinión pública, marca el comienzo de un proceso por el cual dicho concepto que, en principio es bastante impreciso y poco delimitado, se convierte finalmente en un concepto «mensurable». Sin embargo, será precisamente durante la segunda década del siglo $\mathrm{xX}$ cuando se produce un cambio sustancial, tanto en la forma de concebir la opinión pública, como en la metodología que ha de registrarla, cambio que se relaciona con la irrupción de las técnicas cuantitativas procedentes de la psicología, que se están desarrollando en dicha época en Estados Unidos.

\section{EL DESARROLLO METODOLÓGICO: LA CONSTRUCCION DE LA ENCUESTA ESTADISTICA}

A partir del momento en que aparecen los sondeos como técnica de investigación, será necesario un lapso de tiempo muy corto ${ }^{1}$ para que la noción de opinión pública vuelva adaptarse al dispositivo que le confiere objetivación, y pase de ser representado como un concepto ambiguo, con límites poco determinados, a convertirse en un constructo «mensurable».

El cálculo de probabilidades y la estadística constituyen la base -matemática- en la que se sustenta la metodología dominante de la encuesta/sondeo ${ }^{2}$ de opinión pública. En su origen, la estadística se desarrolló a partir de la necesidad de los jefes de estado por conocer los elementos que conformaban su poderío - población, potencia militar, riqueza económica一, para lo cual la idea de registro sistemático y exhaustivo de todos los elementos de la sociedad, encajaba perfectamente con esa necesidad de conocimiento de la mayor calidad y precisión posible. Sin embargo, será a partir de la segunda mitad del siglo XVII

${ }^{1}$ L. Blondiaux estima que bastarán apenas diez años desde la aparición de las encuestas en Estados Unidos, para que se produzca la equiparación entre opinión pública y resultados de los sondeos. (Blodiaux, 1998: 10)

2 A este respecto, sería necesario realizar una precisión lingüística: mientras en castellano los términos encuesta y sondeo (de opinión) han terminado por devenir casi sinónimos en el uso corriente, hay que tener en cuenta que, si bien el francés sondage se aproxima bastante al equivalente en castellano, la palabra enquête tiene, sin embargo, un significado más amplio, más cercano al de «investigación». Así, por ejemplo, es perfectamente válido hablar, de enquête policier, o de enquête par monographie 
cuando surja con fuerza la idea, no sólo de realizar censos que faciliten un registro exhaustivo de datos, sino de conocer y explicar los fenómenos económicos y sociales (Tassi 1988:117-118).

Se trata de una época que coincide con el desarrollo de la aritmética política - precursora de la posterior estadística inferencial-, fundamentalmente en Inglaterra, cuya preocupación principal es la investigación y la cuantificación constante de comportamientos poblacionales que permitan hacer posteriores estimaciones y previsiones ${ }^{3}$. Sin embargo, una serie de cambios tanto desde el punto de vista epistemológico como metodológico, permitirán que hacia la primera mitad del siglo $\mathrm{XIX}^{4}$ pueda hablarse del paso de la aritmética política hasta el nacimiento de la estadística social. Esta transformación se resume en palabras de $\mathrm{M}$. Donnelly, como «el movimiento que va desde la información sobre el cuerpo político (considerando explícitamente las implicaciones del poder del estado) hasta la información sobre el cuerpo social, es decir, la población» (ya no específicamente la comunidad política) (Donnelly, 1996, 230-231).

La gran cantidad de datos numéricos acumulados en series continuas parece haber sido crucial en la aparición de una nueva forma a partir de la cual los estadísticos «contemplan» sus estadísticas, de forma que, a partir de ahora, se comienzan a cuestionar el marco conceptual al cual son referidos los números: «¿son síntomas o indicadores?, ¿qué se cuenta?, ¿ ¿cómo pueden ser interpretados los números?». Y es desde el momento en que tiene lugar el examen concienzudo de las enormes series de datos acumulados, cuando los estadísticos comienzan a «descubrir» regularidades en los mismos; regularidades que les llevan a creer que sus números revelan un nuevo orden de la realidad. Desde nuestro análisis, ¿qué puede suponer este cambio? Podemos decir que aquí comienza un proceso por el cual el número adquiere legitimidad como entidad de objetivación científica, con el poder de revelar «lo que existe», «lo que está ahí fuera» en el terreno de las ciencias sociales; el número será capaz de transformar las meras conjeturas -opiniones ${ }^{5}$ - en hechos científicos, verídicos, y por tanto legítimos para dar cuenta de la realidad.

Este proceso hace que los estadísticos utilicen la noción de regularidad o estabilidad en las proporciones de los fenómenos, para comenzar a hablar de leyes sociales. La idea es la siguiente: la contemplación de los individuos sólo permite ver una cantidad - grande, pero poco definida - de peculiaridades, pero con la agregación de las mismas, las unas se cancelarían con las otras, dejando ver únicamente los rasgos comunes, la generalidad ${ }^{6}$. Esta es la idea que, a través de la aplicación de la distribución normal a la biometría, viene a encarnar el «homme

\footnotetext{
${ }^{3}$ Por ejemplo, el número de hijos por mujer, el tiempo entre dos nacimientos para una misma madre, el número de habitantes por hogar, etc.)

${ }^{4}$ Según M. Donnelly, los historiadores se refieren concretamente al período 1820-1850. (Donelly 1996:230-231)

${ }^{5}$ Entendiéndolas desde la concepción negativa de la que se habló en el apartado anterior.

${ }^{6}$ Esta idea será luego popularizada a través del análisis del suicidio de E. Durkheim.
} 
moyen» de A. Quetelet en su Physique social (Armatte 1995: cap. 7, 42 y ss.), como si la naturaleza se hubiera encargado de producir ese tipo que representa el valor ideal para el grupo, al encarnar los rasgos físicos, morales e intelectuales de toda la sociedad. Esta filosofía, trasladada al terreno de la política, implicará contemplar al elegido como un representante que se constituye como una emanación del grupo, como una opinión colectiva que saldría a la luz sin ser la opinión de ninguno de los miembros individuales.

Es así como A. Quetelet y otros estadísticos coetáneos ayudaron a crear los nuevos hábitos mentales y las nuevas herramientas que construyeron una visión estadística donde el número pasa a ser una forma de representar a toda la población, a la sociedad, como si se produjera una identificación entre lo «justo» en un sentido matemático —en el sentido de precisión-y lo «justo» en un sentido moral. A partir de ese momento, los estadísticos sociales se dedicaron a representar grupos de forma numérica, sin considerar excesivamente necesario hacer referencia a las particularidades y peculiaridades de la cultura, la historia, la lengua y la geografía para dar explicación a los fenómenos sociales (Donelly 1996:236). Estas poderosas técnicas se basarán en la confianza en el agregado, que se considera reflejo o indicador de la esencia real y fundamental de las poblaciones. Así, hay que pensar en la temprana estadística social como un nuevo modo de representación, no exclusivamente como un conjunto de técnicas, sino como un discurso sobre la propia sociedad.

En la época en que aparecen las primeras manifestaciones matemáticas de la historia del cálculo de probabilidades (durante siglos XVI y XVII) el concepto de opinión todavía no había traspasado la barrera «negativa» de lo privado, de forma que hasta mediados del siglo xvill será considerada como un conocimiento provisional (Champagne 1990:45) que implica, al menos un esfuerzo de juicio personal y se sitúa, por tanto, entre la duda y la certidumbre. Si, como señala $\mathrm{E}$. Allo, tomamos en consideración que «el probabilismo presenta el tema de la probabilidad como un grado entre la ignorancia y el saber, y lo sitúa en el interior de un campo subjetivo» (Allo 1984:77-81), no será difícil encontrar la relación entre ambos conceptos ${ }^{7}$, es decir, la opinión como una entidad de la cual hay que calcular su grado de certitud.

Durante los dos siglos que transcurren desde comienzos del siglo XVIII hasta comienzos del XX, encontramos relevantes estadísticos y matemáticos dedicados al cálculo de probabilidades que, influidos también por el contexto político y social de su época se mostrarán interesados en el tratamiento matemático de entidades que no parecen tan fácil ni obviamente «medibles» ni «cuantificables» como las que pueden encontrarse en el mundo físico. Haremos ahora referencia a algunos de los autores cuyas aportaciones desde la matemática van orientadas a dar explicación a fenómenos políticos y sociales, y que se manifiestan fundamentalmente en el notable interés por la aplicación de la estadística y el cálculo

${ }^{7}$ No en vano, señala E. Allo que con el artículo De inceti aestimatione de 1678, Leibniz se estaba refiriendo a la formación de la opinión (Allo, 1984: 79)

EMPIRIA. Revista de Metodología de Ciencias Sociales. N. ${ }^{\circ}$, enero-junio, 2005, pp. 89-113. ISSN: 1139-5737 
de probabilidades a fenómenos tales como los testimonios, las sentencias de un juez, y la toma de decisiones colectivas y asamblearias.

J. Bernouilli ${ }^{8}$, a comienzos del siglo XVIII, procederá a aplicar «la doctrina precedente» (Meusnier 1995:5-28), refiriéndose a lo que posteriormente se denominará «ley de los grandes números» ${ }^{9}$, y que había desarrollado en las tres primeras partes de su Ars Conjectandi, a los asuntos «civiles, morales y económicos». Entiende con la noción de probabilidad, un grado de certitud subjetiva ${ }^{10}$ $\mathrm{y}$, por tanto, cuando habla de ella, se refiere a la probabilidad de una opinión, de la probabilidad de un juicio, admitiendo que ese grado de certitud (de probabilidad) que puede llevar asociada una opinión puede ser medido por el peso de los argumentos que la sostienen:

«El objetivo es que en nuestros juicios y en nuestras acciones pudiéramos siempre elegir o seguir la opción que hemos descubierto como mejor, preferible, más segura o mejor reflexionada» (Armatte 1995:cap. 5,20)

«Las probabilidades son estimadas según el número y también el peso de los argumentos que de alguna manera prueban o revelan que algo es, seráo ha sido» (Armatte 1995:cap. 5,21)

Aplicado al ámbito de los testimonios, admite que la multiplicación de los argumentos no producirá necesariamente una mejora en el grado de certidumbre final, sino que todo dependerá del tipo de argumento de que se trate. Es interesante también comprobar que J. Bernouilli establece una diferencia entre lo que considera un cálculo de probación, que mide el peso de un argumento relativo a un fenómeno, y un cálculo de probabilidad, que mediría la probabilidad de un acontecimiento sin referencia a los argumentos. De ello puede deducirse que una mejora en el grado de certitud, es decir, del grado en que podemos confiar en una opinión, no depende exclusivamente del número de adscripciones, sino también del peso de los argumentos que la sostienen. Ello no parece incoherente con el ya anteriormente mencionado concepto positivo de opinión que cobra fuerza a lo largo del siglo XVIII, basado no el número, sino en la «calidad ilustrada» del grupo social que la sostiene.

Las tesis de J. Bernouilli serán seguidas explícitamente por su sobrino Nicolas ${ }^{11}$ (1687-1759), que las aplica concretamente a cuestiones relacionadas con el derecho, fundamentalmente a los problemas de la herencia y la esperanza de

${ }^{8}$ Jacques Bernouilli (1655-1705) escribe en los albores del siglo XVIII su conocido Ars Conjectandi, obra compuesta por cuatro partes y publicada en 1713, sólo unos pocos años tras su muerte. La parte cuarta «trata del uso y la aplicación de la doctrina precedente a los asuntos civiles, morales y económicos»

9 Dicha ley afirma que la frecuencia de aparición de un fenómeno tiene asociada una probabilidad, y tiende hacia ella conforme aumenta el número de pruebas

10 Relativa al grado de creencia ante fenómenos inciertos, debido al desconocimiento de las leyes que los rigen

11 Nicolas Bernouilli aplicará las ideas de su tío en la tesis doctoral «De usu Artis Conjectandi in Jure» que defiende en 1709 
vida. En el capítulo IX de su tesis ${ }^{12}$ volverá a la problemática del testimonio para aplicar el cálculo de probabilidades al grado de confianza que puede esperarse de un testigo, es decir al cálculo de la probabilidad de que un testigo diga la verdad.

De hecho, la cuestión sobre el grado de confianza en los testimonios será un tema frecuentemente tratado durante el siglo XVIII. Así, Gabriel Cramer (17041752), que conoce muy bien las ideas J. Bernouilli, dedicará gran parte de su obra «Cours de logique» (Cramer, 1740 aprox.: 262-300) a la probabilidad de los testimonios para mostrar que el grado de confianza que se podrá tener en un testimonio dependerá de la cantidad de testigos que concuerden con el mismo, concluyendo así que la probabilidad de la veracidad del testimonio progresa conforme aumenta la cantidad de testigos que la sostienen. Por otra parte, Diderot también desarrollará la misma problemática en algunos artículos publicados en los últimos volúmenes de la Enciclopedia (1765: 393-400) aparecidos en 1765, refiriéndose fundamentalmente a la mayor confianza y, por tanto, mayor probabilidad de aquellos testimonios que adoptan la forma escrita: cuando un testimonio se transmite por escrito, el grado de confianza que podemos tener en su certidumbre - su probabilidad - aumenta infinitamente debido a que las copias impresas hacen que pueda conservarse durante mucho más tiempo, generando otras tantas cadenas que hacen que su probabilidad se acerque infinitamente a la certeza (De Mora 2004:98).

Por su parte, Condorcet (1743-1794), imbuido de la filosofía política imperante en su época y testigo de los acontecimientos revolucionarios ${ }^{13}$, aparte de mostrar interés en la aplicación del cálculo de probabilidades al problema del testimonio, se dedicó también a la cuestión de las decisiones colectivas, manifestando su preocupación por la toma de decisiones por mayoría de votos (De Mora 2004:100 y ss) -cuestión que hizo célebre su famosa paradoja de las mayorías cíclicas ${ }^{14}$ - Así, con el objetivo de llegar a la verdad, intentó encontrar un método para tomar la decisión correcta en el contexto de los tribunales y las elecciones ${ }^{15}$. Condorcet era un gran temeroso del advenimiento de una demo-

12 Titulado «Caput IX. De fide testium \& de suspicionibus; item de commodatoario, an teneatur casum praestare, si res apud commodantem peritura non fuiste, en De Usu Artis conjectandi in Jure» («Chapitre IX: La bonne foi des témoins et les soupçons; le commodataire est-il tenu pour responsable d'une chose qui n'était pas destinée à être perdue par le prêteur?» Trad. de N. Meusnier. (Bernouilli, 1709)

${ }^{13}$ Aparte de su dedicación al cálculo de probabilidades, destacó como hombre político, republicano y partidario de la modificación completa del estado, dada su afinidad con el federalismo y el regionalismo.

${ }_{14}$ A través de esta «paradoja», Condorcet muestra que, en una elección triangular (un cuerpo electoral dividido en tres corrientes de voto), sea cual sea el modo de escrutinio y sea cual sea el candidato elegido, una vez efectuada la votación siempre se puede encontrar una mayoría de electores que estarían dispuestos a votar a uno de los otros dos candidatos derrotados.

15 Estas problemáticas serán desarrolladas en:

- CONDORCET. Essai d'application de l'analyse à la probabilité des decisions rendues à la pluralité des voix. Paris. 1785

- CONDORCET. Essai sur la constitution et la formations des assemblés provinciales. Paris. 1788. 
cracia inculta, es decir, aquella que se encarnaría en la parte de la población cuya opinión era «común», «vulgar», «ignorante». De hecho, mostró su total desconfianza al definir opinión pública como «aquélla de la parte del pueblo más estúpida y más miserable» (Farge 1992:14). Y aunque considera que las decisiones mayoritarias son frecuentemente más correctas que las tomadas por los individuos aislados, sólo si la probabilidad de que cada uno de los individuos que componen una asamblea tome una decisión correcta es superior a la probabilidad de que ocurra lo contrario, puede esperarse que la decisión mayoritaria resultante sea asimismo correcta (Grofman 1989:101-106). De ahí la importancia que para el autor adquiere la educación y la buena información del votante, es decir, que su opinión sea «ilustrada», «culta», capaz de emitir argumentos legítimos; importancia que no es distinta a la que, como se ha visto, adquiere esa connotación de opinión pública, precisamente a finales del siglo XVIII. Finalmente, y de forma coherente con lo anterior, otra de las consecuencia políticas derivadas de su teoría probabilística será la censura que, en su proyecto de Constitución, ejercerá sobre el «pueblo» al separarlo del derecho de sancionar las leyes, excluyéndolo por tanto del poder legislativo para limitar la influencia de lo que consideraba «el populacho»(Pertué 1989: 322-329).

Desde el punto de vista de las opiniones en su relación con las actitudes, es interesante considerar la aportación de A. Quetelet (1796-1894) a la medida de las cualidades morales, fundamentalmente lo que denominaba la inclinación al crimen o al suicidio. Bajo el principio de que «los efectos son proporcionales a las causas que los producen» (Armatte 1995:cap. 7, 59), lo que interesa a Quetelet serán los mecanismos fundamentales que explican las regularidades observadas. Así, considera que el crimen no es más que un efecto de una causa inobservable, a la que denominará inclinación al crimen, y que constituye, como cualquier otra inclinación, cierta característica escondida del individuo típico, o lo que viene a ser lo mismo, de una población homogénea. Como señala M. Armatte, se trataría entonces de una característica tanto individual como colectiva (admitiendo que cada individuo es una copia, una variante ligeramente deformada del tipo) (Armatte 1995: cap. 7,61). No es difícil encontrar paralelismos entre esta noción de inclinación y lo que posteriormente se denominará actitud -como «una predisposición a actuar»- por la psicología social de los años treinta en el entorno norteamericano.

Para continuar este breve recorrido ${ }^{16}$ consideraremos la aportación de E. Borel (1871-1956). Hombre político y situado a la izquierda, fue diputado y siempre se mostró muy interesado por la participación en la vida pública. Así, en «Le

${ }^{16}$ Hay que señalar que otros estadísticos aparte de los mencionados tuvieron importantes contribuciones en el tratamiento probabilístico de los testimonios, las sentencias y las decisiones colectivas. Así, durante el siglo XVIII, J. Borda, académico de las Ciencias, dedicó parte de su obra a la problemática del escrutinio en las elecciones. Ya en el siglo XIX, P. S. Laplace dedicará también parte de su estudio a la probabilidad de la certeza de los testimonios, mientras que S. D. Poisson y A. Cournot se centrarán en la problemática de la aplicación del cálculo de probabilidades a las sentencias judiciales sobre asuntos criminales y civiles. 
calcul des probabilités et la méthode des majorités» (Borel, 1908a: 125-151) desarrollará un procedimiento consistente en:

«...observar como prácticamente válida la opinión expresada por la mayoría, tras el examen de los puntos de vista y las opiniones de un número más o menos grande de personas» ${ }^{17}$.

A lo largo del texto, su propósito será mostrar la utilidad del cálculo de probabilidades para el método de las mayorías, cuestión que aprovechará igualmente para lanzar una crítica - no sin cierta dosis de ironía- hacia aquéllos que, como Stuart Mill ${ }^{18}$, habían manifestado su desaprobación a la hora de aplicar la probabilidad a cuestiones de derecho. La importancia del método de las mayorías reside en que podemos encontrar en él la antesala de lo que será el método representativo, que se desarrollará y usará plenamente en las encuestas estadísticas y en los sondeos de opinión a partir de los años treinta en Estados Unidos. Así, señala Borel:

«El método de las mayorías consistirá entonces, en consultar solamente un pequeño número de personas, con el fin de predecir el resultado que ofrecería el recuento general» ${ }^{19}$.

Lo único que haría falta para que el método funcione es, según Borel, que el grupo total en relación al cual se plantea determinada cuestión sea sensiblemente homogéneo, mientras que el grupo parcial representativo del total ha de aproximarse a la misma heterogeneidad que posee el grupo total. Como puede observarse, Borel hace depender la precisión de la inferencia, no sobre el tamaño del grupo parcial, sino sobre el grado de heterogeneidad - la varianza - del grupo total. No llega mucho más lejos respecto a este punto ${ }^{20} \multimap$ el tamaño que debería tener el grupo representativo y la forma de seleccionarlo- pero sí señala que la relación que existirá entre la verdad relativa (refiriéndose al valor observado) y la verdad absoluta (el valor teórico) depende de un coeficiente de probabilidad que además tiende a la unidad siempre que el grupo parcial sea una imagen fiel del grupo total ${ }^{21}$.

17 Traducido del original: «Nous donnerons, d'une manière générale, le nom de "méthode des majorités» au procédé qui consiste à regarder comme pratiquement valable l'avis exprimé par la majorité, après dépouillement des avis ou des opinions d' un nombre plus ou moins grand de personnes" (Borel 1908a: 125)

${ }_{18}$ Stuart Mill había señalado que la aplicación del cálculo de probabilidades a las decisiones judiciales constituía «el escándalo de las Matemáticas». (Citado por Borel, 1908a)

${ }_{19}$ Traducido del original: «La méthode des majorités consistera donc, en consultant un petit nombre seulement de personnes, à chercher à prevoir le résultat que donnerait le dénombrement général» (Borel, 1908a: 129)

${ }^{20}$ Hay que señalar que en esa época se está desarrollando el debate sobre el método representativo en el seno de los congresos sucesivos del Instituto Internacional de Estadística. Dos años antes del artículo de E. Borel, A. Bowley ya había introducido el cálculo de los intervalos de confianza.

${ }^{21}$ Cuestion en la que Borel mostraba mucha confianza. (Borel, 1908a: 130-131) 
En otro artículo publicado durante la misma época (Borel, 1908b) en la revista que él mismo crea, Revue de Mois, y dado su interés por las cuestiones de psicología, hace un análisis de las críticas que hasta el momento había recibido la estadística, provenientes fundamentalmente de lo que llama la «sensibilidad individualista»(Borel, 1908b: 642). Estas críticas giran en torno al temor -sobre todo por parte de las élites- a la sensación de pérdida de individualidad y unicidad que conlleva el ser «tomado por un número», a la pretensión de prever en cierta medida los acontecimientos futuros y a su correlato de sentimiento de pérdida de la libertad individual. Borel responderá a esta corriente individualista destacando que el cálculo de probabilidades se encuentra en el fundamento de las matemáticas sociales (Borel, 1908b: 650-651): ellas nos recuerdan que los seres humanos vivimos en sociedad y que los fenómenos sociales tienen una existencia real y un interés propio. Y, curiosamente, termina viendo en la aplicación del número a los fenómenos sociales una serie de ventajas que trascenderían el ámbito de lo puramente metodológico y se acercan al terreno de la moral, atribuyendo al cálculo de probabilidades un alto «valor educativo» capaz de combatir ese cierto individualismo, que no sería otra cosa que egoísmo «poco inteligente»:

«El estudio de estos hechos no puede más que contribuir al desarrollo de la noción de solidaridad, a recordar a cada cual que no debe considerarse como independiente del medio donde vive y que debe participar en la reparación de los daños fortuitos que alcanzan a su vecino y podrían alcanzarle a él mismo» (Borel, 1908b: 651)

Pero a lo largo de su andadura, la estadística no ha sido considerada únicamente por sus usos como una herramienta metodológica. Así, el estadístico alemán E. J. Gumbel (1891-1966), desde una posición marxista, trata de vincular las matemáticas con la acción política: el interés creciente suscitado por la Unión Soviética de principios de los años veinte y su activismo a favor de la causa pacifista y contra el nacional-socialismo alemán, se encuentran muy relacionados con su motivación explícita de mostrar el rol decisivo que la estadística matemática sería capaz de ejercer en una economía socialista planificada (Hertz 2000:163-187). Ello le llevará a escribir un manifiesto «Estadística y lucha de clases ${ }^{22}$ que aparece en 1928, donde tras reconocer que la estadística es una ciencia específica del capitalismo adaptada de forma habitual a la economía política burguesa, afirma que igualmente es posible sostener otra concepción distinta de la disciplina que puede ser útil y necesaria a la causa del proletariado.

Así, considera que la estadística sólo se ha convertido en una herramienta necesaria -en la forma «actual»- cuando el capitalismo ha permitido la emergencia de unos estados bien establecidos que cuentan con un aparato burocrático que hacen uso de ella ordinariamente. Sin embargo, esa misma estadística parece suponer para el autor un arma de doble filo al poder ser utilizada, a la vez,

22 Texto original: Klassenkampf und Statistik(Gumbel, 1928). 
para examinar teóricamente, desde un punto de vista crítico, el proceso de producción, distribución y consumo en el propio seno del sistema capitalista dominante. Sin embargo, desde el punto de vista metodológico, encuentra en la encuesta ${ }^{23}$ una serie de límites naturales, al reconocer que sólo pueden ser objeto de la estadística los hechos simples, es decir, aquellos de los cuales la respuesta del encuestado puede ser emitida sin dificultad. No así el caso de la investigación de las causas, sobre todo en lo que respecta a los fenómenos subjetivos, fundamentalmente aquellos cuya divulgación puede parecer inoportuna para el encuestado. Reconoce Gumbel que, en este caso, no sólo se trata de un condicionamiento natural, sino que existen condicionamientos sociales y psicológicos (grado de aquiescencia hacia la estadística, nivel de educación, opinión hacia la persona que interroga) que median en la calidad de la respuesta obtenida por el encuestado durante el momento de interacción con el entrevistador. Es así como el autor, dando la vuelta al argumento, justificará - y legitimará- el uso de la estadística en el seno de una colectividad inmersa en una economía planificada:

«Una comunidad con un alto nivel de educación, donde predomine la consciencia de que el gobierno se ocupa del interés de la mayoría de la población, podrá, e incluso deberá, plantear a sus miembros otro tipo de cuestiones, además constructivas» (Gumbel 1928:190).

En realidad, parece que Gumbel sólo encontraría un verdadero uso de la estadística para el estudio de «hechos constatados», prescindiendo más bien de los elementos subjetivos -opiniones, motivos, actitudes-. Es decir, plantea el registro de elementos distintos a los que toma en consideración la estadística burguesa, pero siempre en el terreno de los hechos «objetivos».

Ya desde la sociología propiamente dicha, concluiremos este viaje con la referencia a Maurice Halbwachs (1877-1945), sociólogo francés que, durante los años en que se está produciendo el arranque de los sondeos como método de investigación en las ciencias sociales, desarrollará una visión crítica respecto a los usos de la estadística y las matemáticas en el estudio de los fenómenos sociales. Si bien su tesis sobre «La clase obrera y los niveles de vida» (1912), apoyada de forma determinante sobre el análisis de datos procedentes de estadísticas alemanas, no pone en duda ni el sobrado interés ni la formación estadística con que cuenta el autor (Arribas 2004:338-342), hay que reconocer en él una mirada «atenta» al rol que la estadística y las matemáticas pueden desempeñar en sociología (Martin 1999:69-101).

Así lo manifestará en el texto «La statistique, ses aplications et les problèmes qu'elles soulèvent» (1935) presentado a propósito de su intervención en un coloquio de la «semaine de synthèse», donde además de intentar mostrar el poder de la estadística y las matemáticas como forma de reconocer las regularidades so-

${ }^{23}$ He traducido «encuesta» del francés «enquête» por considerar este sentido como el más próximo al que puede referirse Gumbel en su texto original, pero sin obviar las precauciones que sobre dicha traducción se comentaron anteriormente (ver nota al pie $n .{ }^{\circ} 2$ ). 
ciales, señalará también los riesgos que ambas pueden suponer en su aplicación a las ciencias sociales. Dichas regularidades son la manifestación de lo colectivo, de la cohesión de los fenómenos sociales, y no de la combinación de múltiples constantes y variables independientes las unas de las otras. Por tanto, si para Halbwachs, la principal virtud de la estadística consiste en apreciar los rasgos del colectivo, es decir, mostrar las características de un grupo que no pueden ser descubiertas en ningún miembro del mismo tomado aisladamente, hay que tener igualmente la precaución de considerar los análisis estadísticos, no como fines en sí mismos de la investigación, sino como «instrumentos de observación y de comparación, precisos y objetivos » ${ }^{24} \mathrm{y}$, por tanto imprescindibles que, sin embargo, «no nos aportan teorías». Así, la estadística aportaría la materia prima -muy valiosa, eso sí- de un posterior análisis sociológico profundo que permite comprender la lógica social presente en los resultados estadísticos.

Con ello Halbwachs mostrará una posición crítica frente a los excesos de la estandarización y la abstracción que comenzaban a tener lugar con la aplicación de las técnicas cuantitativas a las ciencias sociales (Arribas 2004:339). Así, se opone tanto al estudio de estadísticas sobre grupos o conjuntos de gran tamaño que, de hecho, esconden realidades muy diversas, como a la extracción de la realidad social de un pequeño número de hechos perfectamente circunscritos y sacados así de su contexto. Todo ello no hace más que separar al sociólogo de su verdadero objetivo, que es el estudio de las diferencias ${ }^{25}$. No es posible extraer de su contexto los acontecimientos humanos, pues los hombres se encuentran insertos en la sociedad, y se definen en relación a ella. De ahí, que su concepción de investigación social excluya la realización de encuestas de un gran número de casos simples —que él califica de extensivas — por disimular y esconder errores e influencias de los casos excepcionales, al igual que el método intensivo de Le Play, cuya observación de «casos típicos» juzgados como casos «medios», «se corre el riesgo de truncar la realidad».

Por tanto, podemos concluir que, para Halbwachs, las matemáticas constituyen un instrumento metodológico imprescindible para el investigador social que permite aproximarle a regularidades sobre los fenómenos sociales a las que no podría acceder de otra forma, si bien, no sustituyen al verdadero trabajo de interpretación que permite al sociólogo conocer la verdadera «naturaleza» de los fenómenos sociales.

Una vez realizado este breve repaso a las aportaciones de los estadísticos que, antes de la eclosión de los sondeos de opinión, intentaron acercarse al tratamiento matemático de los fenómenos políticos y sociales, el interés reside en saber cómo se introduce y qué visiones del mundo deja implícitamente percibir el verdadero sustento metodológico que permite el desarrollo de las encuestas de opinión: el método representativo. De ello nos ocuparemos a continuación.

${ }^{24}$ Citado en Martin, 1999: 77

${ }^{25}$ De ahí que mantenga igualmente una postura crítica frente a la teoría del hombre medio Quetelet. (Martin, 1999: 85-86) 


\section{LA INTRODUCCIÓN DEL METODO REPRESENTATIVO}

El paso previo a la proliferación de las encuestas estadísticas que, tanto sobre opinión publica como sobre otras cuestiones, tendrá lugar a partir de los años treinta, consiste en encontrar un método que permita «legítimamente» registrar información, no de todos y cada unos de los miembros de la población objetivo de estudio, pues ello supone, además de la complejidad del procedimiento, unos costes inasumibles en términos económicos y temporales, sino solamente de una parte de la misma. Sin embargo, aunque el concepto de representatividad que subyace a las encuestas estadísticas tal y como se llevan a cabo hoy en día, es el que se tiene por asumido como «evidente», como «válido», es posible encontrar lógicas diferentes a la hora de entender la dicho concepto. De hecho, las distintas formas de entender, en la práctica, el método representativo han ido evolucionando paralelamente a las formas de entender la noción de representatividad - y por ende, de la parte y del todo- en el transcurso del debate metodológico que sirve de contexto para que los estadísticos vayan configurando el método en lo que será finalmente su forma definitiva.

Se ha apuntado como antecesor del método representativo las técnicas del multiplicador de nacimientos ${ }^{26}$ procedentes de la aritmética política inglesa que se estaban desarrollando a partir de la segunda mitad del siglo XVII (Tassi 1988:118). Sin embargo, el debate sobre el método representativo en sí mismo es bastante reciente, puesto que data de finales del siglo XIX, cuando el noruego Kiaer lo introduce en el seno del Congreso del Instituto Internacional de Estadística celebrado en su país en 1895 (Desrosières 2004:246-247). A partir de entonces, los congresos celebrados hasta los años treinta van a ser el contexto en el que se desarrolla un debate donde, paulatinamente se irán abordando tres cuestiones relativas al método representativo. En un primer momento se discute sobre la legitimidad del método en sí como posible forma de obtener información de la población sin tener que recurrir a censos exhaustivos, a la vez que, paralelamente, se debate su pertinencia como «mejor» forma de proceder en comparación con el método de las monografías que todavía tenían en la época un peso muy importante. Finalmente, una vez legitimado el sondeo como método, el debate girará en torno a la posibilidad de incluir el azar como forma de seleccionar la muestra representativa, es decir, a la elección entre el muestreo aleatorio y la selección intencional ${ }^{27}$.

Sin entrar en el debate metodológico en sí ${ }^{28}$, interesa destacar las descripciones del mundo social que tal debate deja entrever. En un primer momento, la propuesta de un método que sustituyera la exhaustividad de un censo se enfrentó a numerosas críticas, pues sólo se concebía como legítima desde el punto de vista metodológico esa última forma de proceder. Sin embargo, como se dijo ante-

\footnotetext{
${ }^{26}$ Relación entre la población y los nacimientos.

${ }^{27}$ En francés se utilizará el término «choix judicieux» 0 «choix raisonné» para referirse a la «selección intencional».

28 Ver Tassi, $1988: 117-133$.
} 
riormente, criterios de economicidad recomendaban proceder de otra forma, incluso sabiendo que la información que el método representativo podía aportar iba a ser «imperfecta». Esta idea es la que expresa Karl Mayer —con cierta resignación-al apoyar la propuesta de Kiaer en el congreso del IIE celebrado en 1903:

«...el conocimiento aproximadamente justo obtenido por una estimación sistemática es mejor que la falta completa de conocimientos...» (Tassi 1988:122).

Pero respecto a la competencia entre dos metodologías diferentes, sondeos y monografias, vemos que ambas responden a una forma distinta de entender la representatividad; tampoco el todo y la parte se consideran de la misma forma. Las monografías que se habían desarrollado durante el siglo XIX, fundamentalmente de la mano de Le Play, tratan de ofrecer una análisis comprensivo, basado en la búsqueda de «casos típicos», donde la «media», la «regularidad», encarnarían la totalidad (Desrosières 2004:234-238). Es fácil ver cómo esta concepción corresponde al esquema ya visto de Quetelet: para él, el «hombre medio» ya representa y resume en sí mismo a toda la población, en la cual, las desviaciones son «imperfecciones» respecto de ese modelo ideal. Sin embargo, a partir de la propuesta de Kiaer ${ }^{29}$, el todo que sirve de referente tiene una estructura, tiene una diversidad interna de la que es necesario dar cuenta, si queremos que la parte sea verdaderamente representativa. Así, vemos como la representatividad se entiende desde un punto de vista totalmente nuevo.

Las herramientas técnicas que den soporte metodológico a esta nueva forma de entender la representatividad van a llegar fundamentalmente en dos momentos: primero, en 1906 el inglés A. Bowley introducirá a través su comunicación en la Royal Statistical Society y al margen del IIE, el cálculo de los intervalos de confianza, que permiten tener una medida del grado de representatividad de la muestra y, en segundo lugar, Neyman introduce en 1934 el muestreo estratificado con afijación óptima y el muestreo en dos etapas en $1938^{30}$, dejando así zanjada la polémica entre selección intencional y selección aleatoria. A partir de ahora interesa calcular varianzas, regresiones, correlaciones, es decir, interesa establecer comparaciones entre los individuos.

Sin embargo, el hecho de que Neyman pusiera fin a una polémica no significa que haya sido el primero en introducir tales métodos. Como señala $M$. Mespoulet ${ }^{31}$, las técnicas de muestreo han sido utilizadas por los estadísticos rusos desde fechas muy tempranas. De hecho, el estadístico Kovalevskii introdujo

29 A pesar de hablar de representatividad, todavía no dispone de las herramientas metodologicas que le permitirán su tratamiento estadístico

${ }^{30}$ Así, Neyman responde a C. Gini y a L.Galvani, que habían utilizado la selección intencional: «un método de sondeos será representativo si permite obtener estimadores convergentes y calcular la precisión de los resultados». Citado por Tassi, 1998: 126.

${ }^{31}$ Mespoulet, M. La edad de oro del sondeo en Rusia (1885-1924). Seminario de Estadística y Ciencias Sociales. Departamento de Sociología I (Teoría Metodología y Cambio Social). Facultad de Ciencias Políticas y Sociología (UNED). Madrid, 26 marzo, 2004 
el método de estratificación con afijación óptima diez años antes de que lo hiciera Neyman. Sin duda, las dificultades debidas al alejamiento espacial y lingüístico y los acontecimientos ligados a la revolución de octubre obstaculizaron el conocimiento de la escuela rusa dedicada a la «estadística parcial».

Como apunta P. Tassi, las «ironías del tiempo» harán que la crítica que hace Neyman a la selección intencional vaya a coincidir precisamente con el momento en que se produce la emergencia de los sondeos de opinión (Tassi 1988:126), los cuales se valdrán al comienzo de su andadura, de esa misma técnica o, como mucho, de una de sus variantes, el muestro por cuotas.

\section{EL TRIUNFO DEL MÉTODO: EL MONOPOLIO DE LOS SONDEOS DE OPINIÓN}

La emergencia de los sondeos de opinión va a tener lugar en un contexto de cambios sociales. En la Norteamérica de los años veinte, la formación de la llamada sociedad de consumo de masas junto a la irrupción de los medios de comunicación, igualmente de masas que se encargan de dar cobertura a los procesos electorales, había generado un nuevo campo de investigación social (Camarero 2001:171), donde se va pasar del interés por el muestreo con el fin de obtener información fundamentalmente para el Estado, hasta la pretensión de los periodistas por «pulsar la opinión» sin ninguna preocupación por la validez metodológica ni la representatividad.

Así, puede considerarse como verdadero origen de los sondeos de opinión ${ }^{32}$ la cobertura de las elecciones presidenciales que, desde 1824, viene realizándose en Estados Unidos a través de los llamados «votos de paja» y que será una práctica habitual de diversos periódicos estadounidenses, como New York $\mathrm{He}$ rald, Chicago American, Columbus Dispatch y, muy especialmente a partir de 1916, The Literary Digest. Se trata de muestras enormemente elevadas - en relación a la práctica habitual hoy en día- que no reposan sobre ningún criterio de representatividad estadístico.

El arranque de los sondeos se encuentra en la encrucijada de dos procesos que van a encontrar su proceso de maduración durante la segunda y tercera década del siglo $\mathrm{XX}$, y que van a ser fundamentales para la consolidación, a partir de la Segunda Guerra Mundial, de las encuestas de opinión como dispositivo legítimo de investigación de la opinión publica. Por un lado, desde el punto de vis-

${ }^{32}$ A este respecto, mucho se ha escrito sobre el origen "primero" de los sondeos de opinión. Así, señala P. Beaud que la primera descripción de un sondeo de opinión nos la ha dado Tucídides en su «Historia de al guerra entre les peloponesos y les atenienses»: los lacedemonios tenían la costumbre de pronunciarse sobre los asuntos públicos a través de aclamaciones, pero con motivo de la guerra con los atenienses, el magistrado elegido fue incapaz de discernir tras el debate qué posición era la más fuerte, así que decidió contar las opiniones: «quienes de entre vosotros, crean que el tratado es roto y que los atenienses son culpables se levanten y vengan a agruparse a este lado, y los que son de la opinión contraria, del otro lado". Entonces el tratado fue roto con una fuerte mayoría. (Beaud y Quere 1990:35) 
ta metodológico, la encuesta estadística basada en muestras representativas acaba de encontrar, gracias a las aportaciones de Neyman, el fundamento matemático (y por tanto, científico) necesario como para ser considerada una técnica «madura»; por otro lado, el interés por la medición de actitudes se acompaña del desarrollo de herramientas matemáticas análogas a las que los psicólogos aplican para medir el coeficiente de inteligencia de Binet ${ }^{33}$. La convergencia de ambos procesos favorece enormemente una forma de entender la investigación social que constituirá la especificidad de la tradición sociológica norteamericana, y que se ha dado en llamar positivismo instrumental o empirismo abstracto. Esta nueva forma implica la concepción de un modelo de sociedad atomista o individualista, donde la teorización engloba la definición de conceptos en términos de los procedimientos de medida y en la elaboración de hipótesis contrastables empíricamente (Scheweber 2002:66 y ss.). Ahora, la medida se convierte en una precondición para la definición de conceptos.

El hecho simbólico - y mítico- que terminó por legitimar, ya no solamente desde los círculos intelectuales y científicos, sino de cara al público general, la técnica de la encuesta estadística basada en muestras representativas, fue el «golpe» asestado por G. Gallup a los «votos de paja» con motivo de la celebración de las elecciones presidenciales norteamericanas de 1936. El sondeo realizado por Gallup ${ }^{34}$ utilizando el método representativo, fue capaz de predecir la victoria de Roosevelt con un mínimo error, en contraste con la equivocación en los resultados del «sondeo de paja» realizado por The Literary Digest que, con una muestra mucho mayor que, sin embargo, no seguía ningún criterio de representatividad estadística ${ }^{35}$, se inclinó por anunciar la victoria del candidato republicano. Es a partir de este momento cuando se va a producir una asimilación entre sondeo y opinión, entre sampling y pooling, entre lo que se ha ido construyendo como una innovación metodológica —el uso de la muestra representativa-y lo que era el objeto de «medida» - la opinión- (Armatte 2003:231).

Desde entonces es extremadamente rápido el proceso por el que los distintos grupos sociales (periodistas, universitarios, grupos comerciales, responsables del gobierno) van a apropiarse de la técnica del sondeo. El primer fracaso sonado de los «sondeadores» Gallup y Roper en la predicción de Dewey como candidato ganador en las elecciones presidenciales de 1948, tendrá, sin embargo, mínimas consecuencias a nivel material y simbólico: continúa la colaboración institucional entre institutos de sondeos y universidad, y tampoco se pierde la clientela for-

${ }^{33}$ A pesar de que, como señala $O$. Martin «Binet y Simon excluyen que su escala métrica de la inteligencia permita medir, en el sentido matemático o físico, la inteligencia. Permite simplemente establecer una clasificación, una jerarquía, pero no una medida» (Martin 1997: 34).

${ }^{34}$ También A. Crossley y E. Roper, habían realizado sondeos representativos con resultados similares al que Gallup llevó a cabo. Los tres utilizaron muestras que oscilaban entre 4000 y 5000 personas (que, de dotas formas, son ampliamente superiores a las que se llevan a cabo en la actualidad para los mismos fines)

${ }^{35}$ La muestra estaba compuesta por alrededor de 2.400 .000 personas, pero el criterio para su formación consistía en incluir a los suscriptores de la revista y a los propietarios de vehículos, lo cual, obviamente, provocó un sesgo en favor del candidato republicano Alf Landon 
mada por la prensa. Así, los sondeos de opinión se presentan como un dispositivo sólido, capaz de soportar las consecuencias de esta «revelación de debilidad» (Blondiaux 1998:277-278). A este respecto, J. Stoetzel, introductor de los sondeos de opinión en Francia, señala:

«Los sondeos, se dirá, que sólo han reportado éxitos. Si el de 1936 acuña, de alguna forma, su acto de nacimiento, el «fracaso» de 1948 en circunstancias análogas, momento de la elección del Presidente Truman, revela su fragilidad (...) Al final ¿qué ciencia no tiene una historia jalonada por fracasos? Un avión que se cae no condena la aviación. Es más, las dificultades y los fracasos estimulan el espíritu de investigación, y contribuyen a menudo al perfeccionamiento de las técnicas y al progreso de la teoria" (Stoetzel, Girard 1973:53).

Así, parece que el episodio de 1948, en lugar de plantear el uso de la técnica, ha quedado como esa «excepción que confirma la regla», es decir, como ese dispositivo sólido y fiable al que se permite, como puede esperarse, tener algún que otro «traspiés».

Si se ha de señalar otro importante momento que consolida la autoridad metodológica de la encuesta estadística fue la publicación por Samuel Stouffer de la investigación «The American Soldier» (Stouffer, 1949), que va aparecer en un contexto dominado por el patronazgo militar y gubernamental de las técnicas cuantitativas, por el desarrollo de las ciencias conductistas -que combinan la antropología cultural, la psicología social y la sociología-, y por la creación de centros universitarios interdisciplinares dedicados a la ciencia social aplicada. En esta obra se desarrollará una aproximación a la ciencia social basada en la combinación de análisis cuantitativo con encuestas de actitudes a través de escalas de medida, todo ello con el fin de producir un conocimiento científica y políticamente relevante. A partir de ahora el estudio de encuesta irá indisolublemente unido al análisis estadístico, hasta el punto en que tras la guerra se van a considerar como prácticamente sinónimos (Scheweber 2002:65-67).

El traspaso de toda esta corriente metodológica a Europa, concretamente a Francia, llegará de la mano de Jean Stoetzel (1910-1987) que, como se ha dicho, se ha considerado como el padre fundador de la industria del sondeo en tal país y, además, será, sin duda, la persona más influyente de la sociología francesa de postguerra. Desde siempre había mostrado un gran interés por los métodos estadísticos que se venían desarrollando en el ámbito de la psicología, de ahí que emprenda una estancia en la universidad de Columbia durante el período 1937-1938 (Martin, Vannier 2002:108-113), donde va a descubrir las investigaciones norteamericanas sobre la psicología de actitudes y opiniones, y sobre la metodología de construcción de escalas.

La proximidad conceptual - asumida - entre los conceptos de aptitud y de actitud hará que no sea demasiado difícil para los psicólogos aplicar los aparatos metodológicos creados para las aptitudes al terreno de las actitudes: tests/pre- 
gunta-respuesta y escalas. Y, tal como se comento anteriormente, de ahí a la opinión sólo hay un paso más: considerarla como la manifestación verbal de una posición psicológica subyacente, es decir, de una actitud.

Con tal esquema teórico va a fundar Stoetzel en noviembre de 1938, tras su regreso a Francia, el Instituto Francés de la Opinión Pública (IFOP) ${ }^{36}$, lo cual supone para tal país, no sólo el comienzo de un nuevo método de investigación empírica, sino también una nueva forma de pensar la sociedad que Stoetzel trae de Norteamérica basada en el positivismo instrumental. Al año siguiente crea la revista Sondages, en cuyo primer número (de junio de 1939) se expondrán los objetivos que guían al IFOP:

«... la tarea consiste en medir, según las reglas de una metodología rigurosa, la opinión pública. Hemos pensado que esta nueva fuente de informaciones, no sobre los hechos concretos, que llamamos acontecimientos, sino sobre la reacción en el espíritu público de esos acontecimientos, aportará en medio de nuestra época turbulenta, esta clase de seguridad intelectual que hace nacer el conocimiento, aunque imperfecto, de la verdad». (Riffault 1981:232).

Como ocurre con los primeros sondeadores en Estados Unidos, Stoetzel tratará de dar legitimidad a su instrumento a la vista de distintos sistemas de justificación —epistemológicos, políticos, industriales-. Así, con la intención de convertir el IFOP en un instituto similar al fundado por Gallup, se propone dotarlo de los medios necesarios para convertirlo en una organización de «tipo industrial», en la creencia de que las ciencias sociales no deben limitarse a los «métodos artesanales». De ahí que proponga una «organización científica del trabajo» dentro del propio instituto justificándolo como forma de redundar en la calidad de la investigación. Y aunque en su declaración de intenciones figura la pretensión de independencia respecto a otro tipo de fuerzas o influencias, lo cierto es que la necesidad de financiación conduce finalmente a la creación de una «sociedad hermana» del IFOP, -ETMARdedicada a realizar estudios de mercado, y que asume por objetivo «la venta a los industriales de los estudios necesario para la puesta en marcha de políticas de marketing»(Riffault 1981:243). A partir de los años cincuenta, la financiación estará asegurada por contratos de investigación que parten de administraciones y sociedades industriales.

El IFOP adoptará, así, un rol innovador en cuanto a la penetración de las nuevas técnicas (y la nueva filosofía de trabajo) procedentes del cuantitativismo norteamericano. Durante casi tres décadas estuvo funcionando en solitario, hasta que a partir de 1965 aparecen otros institutos de investigación de la opinión pública que, como en el caso de SOFRES ${ }^{37}$, también van a desarrollar sus propias publicaciones (L'état de l'opinion).

${ }^{36}$ Institut Français d'opinion publique (IFOP) se mantiene en activo desde 1938 a 1978

${ }^{37}$ Desde 1987 SOFRES saca a la venta una publicación anual, «L'état de l'opinion» basada en la realización de encuestas sobre diversos temas de opinión pública. 
Así, la técnica de los sondeos de opinión basados en la encuesta estadística, terminará por configurarse como el nuevo dispositivo de objetivación de la opinión pública, y sus resultados serán tomados como la nueva realidad de la opinión ${ }^{38}$, capaz de emitir, gracias a la metodología cuantitativa en que se sustenta, un punto de vista imparcial, neutro, científico y desinteresado sobre la realidad política y social. Como resultado de todo ello, se llegará a una cuasi-identificación entre el concepto de opinión pública y los resultados de los sondeos que perdurará hasta nuestros días, aún a pesar de la corriente crítica que se iniciará a finales de los años sesenta.

La mayor fortuna de la encuesta estadística para imponerse como metodología de investigación de la opinión pública está, por tanto, muy relacionada con el proceso por el cual se dota de «objetividad» científica, y por tanto de neutralidad «no sospechosa» de ningún interés particular a todo aquello que se convierte en mensurable y medible, aquello que se acompaña de una cantidad numérica. Tal como señala T. M. Porter, la objetividad científica proporciona una respuesta a la necesidad «moral» de «imparcialidad», pero teniendo en cuenta que el proceso de objetivación consiste, sobre todo, en la capacidad o la habilidad de llegar a un consenso sobre lo que es objetivo, verdadero (Porter 1995:7387); en definitiva, la cuantificación, dada su capacidad para construir «objetividad», constituye una forma de tomar decisiones, pero sin dar la impresión de que en realidad se está, de hecho, decidiendo.

La cuestión fundamental reside en que el fundamento matemático en el que se basa la técnica de encuesta, la estadística, se ha convertido en una herramienta tan familiar, con unas rutinas y formas de uso tan introducidas en lo cotidiano, que puede decirse que su presencia ha pasado a formar parte del orden de «lo dado por supuesto». Ha logrado ser representada como una herramienta «conveniente», como un conjunto de técnicas «neutras» y «altamente formalizadas», de forma que se tiende a olvidar los contextos sociales en los que ha surgido el trabajo de los estadísticos. M. Donnelly lo expresa perfectamente cuando señala que «hay una considerable ironía histórica cuando se piensa en la estadística como una técnica refinada y pura».(Donnelly 1996:226)

Además de ello, la forma que adopta el pensamiento estadístico ha sido implantada en -y ha impregnado, por ello- «una gran maquinaria burocrática» ${ }^{39}$ que, además de su supuesta única función como proveedora de información, se constituye igualmente como una tecnología del poder en los estados modernos.

De esta forma, los desarrollos logrados por las nuevas técnicas de encuestación unidos al progreso de una teoría orientada a interpretar el crecimiento y desarrollo económico-social alcanzado en la sociedad norteamericana de los años treinta, harán que la nueva metodología encaje perfectamente con la visión y los objetivos de las grandes corporaciones industriales y los poderes fácticos del país.

${ }^{38}$ En el sentido de una realidad concreta, casi física.

${ }^{39}$ Hacking, I. How Should We Do the History o Statistics? I and C (formerly Ideology and Consciousness) 1981. Citado por Donnelly, 1996: 227. 


\section{CONCLUSIÓN: UNA BREVE REFLEXIÓN SOBRE LA METODOLOGÍA}

Desde que aparece la nueva forma de concebir la idea de población procedente de la temprana estadística social, consistente en considerar la población como un sistema que podía ser estudiado como conjunto a través de frecuencias, la institucionalización de la nueva sociología funcionalista, que cobrará un impulso importantísimo en el contexto de los Estados Unidos tras la Segunda Guerra Mundial, hará que, a nivel empírico, la consolidación del empirismo abstracto encuentre todo un campo de aplicación y legitimación en el uso de las encuestas de opinión como mecanismo de diagnóstico y predicción del comportamiento colectivo de la sociedad de consumo de masas que se encuentra, en este contexto espacio-temporal, en pleno apogeo. Pero ello no quiere decir que, a la vez, no hayan surgido voces críticas respecto a la validez de tal metodología. En particular, la profusa publicación de encuestas de opinión en los medios de comunicación de masas, fundamentalmente cuando se trata de períodos electorales, va a evidenciar que, tanto desde el punto de vista teórico como metodológico, la técnica no está exenta de problemas.

Obviamente, la «opinión pública» que emerge de las encuestas no procede, por lo general de un público que ha tenido la oportunidad de formarse una opinión procedente de la discusión, de la puesta en común de los puntos de vista y de un consenso que finalmente pueda suponer una participación activa en la toma de decisiones. Ello no quiere decir que, desde el ámbito de la metodología, no se hayan planteado otras alternativas que resultan menos estrechas de cara a fomentar la participación ${ }^{40}$. Así, muchos autores defienden la metodología cualitativa como forma más adecuada de acercarse al estudio de las opiniones. Así, J. Ibáñez (Almazán, Villarejo 1998:313), y toda una escuela de sociología crítica en España, desde un análisis crítico de la técnica de encuesta para la investigación de mercados, plantearon alternativas concretas: el grupo de discusión, capaz de estudiar fenómenos sociales con profundidad desde una perspectiva holística, y donde prima el concepto de representación social en lugar de hablar de opinión pública. Como señala J. M. Arribas, con frecuencia el investigador se detiene en la explicación minuciosa y detallada de los más complejos procesos de transformación de variables, sin haber dado la más mínima explicación sobre la cualidad o pertinencia de las categorías que va a utilizar en el análisis, y olvidando la relevancia o irrelevancia de los resultados. Así, reivindica la perspectiva cualitativa como forma de aportar una «sensibilidad diferente» a la investigación sociológica cuantitativa, bajo el supuesto de que la «cualidad» precede a la «cantidad» (Arribas 1998:93-94).

En este sentido son interesantes ciertas iniciativas que, institucionalmente, tratan de fomentar espacios en los que exista una mayor posibilidad de generar

${ }^{40}$ Ello a pesar de que, como señala F. Bouza, la potencia y extensión de la tendencia a identificar encuestas y "opinión pública» es fuerte incluso entre sus críticos. (Ver BOUZA, F., Desventuras de un concepto claro y distinto: Opinión Pública, en http://www.ucm.es//info//socvi/BOUZA/NUEVA1/Textos/). 
debate en la sociedad. Es el caso de los «fórums híbridos» que, teniendo en cuenta la distribución desigual de «saberes» (de expertos y profanos), intentan establecer un intercambio de ideas sobre temas que afectan a la colectividad, de forma que las decisiones que potencialmente pueden surgir de tal debate, incorporan algún tipo de consenso entre las partes.

Para concluir, diremos que el concepto de opinión pública sigue estando lejos de la univocidad y de la universalidad. Pero sí que es cierto que una cierta forma de concebir el «público» se acerca más a un concepto - desde luego más normativo que descriptivo- de opinión pública real. Por otra parte, la opinión también ha pasado de constituir una «doxa», es decir, una creencia más o menos fundada, de la que había que estudiar su probabilidad de certidumbre - de ello se encargaban los primeros probabilistas y estadísticos que surgen con el ansia de traducir en números y cifras los fenómenos sociales - hasta una predisposición interna, latente, relacionada con la actitud que solamente tiene capacidad de hacerse pública y objetiva cuando se exterioriza a través de las reacciones de los individuos portadores de actitudes hacia los estímulos que suponen las preguntas de un cuestionario de encuesta. El sondeo y el método representativo en que se basa, tiene verdaderas dificultades para dar cuenta de una entidad que, a priori, no puede medirse como el peso o la talla, o aquéllas medidas que utilizaba Quetelet para dar a luz a su «hombre medio». Y es que pasar de éste a la «opinión media» es bastante complicado.

Así, si se quiere realmente dar una definición de opinión pública, hay que implicarse en el concepto de lo "público», un conjunto de personas con posibilidad para discutir, debatir y poner en común los asuntos que les afectan, y cuya opinión no sea meramente registrada para ser sumada y expuesta en los medios de comunicación de masas, contribuyendo así al proceso de legitimación de la técnica -y del poder-.

En definitiva, explorar y experimentar nuevas perspectivas metodológicas más democráticas y participativas con el fin de que los ciudadanos sean una parte activa en la toma de decisiones. 


\section{BIBLIOGRAFÍA}

Allo, E. (1984): L'émergence des probabilités. Actes de la Recherche en Sciences Sociales, n. ${ }^{\circ}$ 54. Sept., pp. 77-81.

AlmazÁn Llorente, A. y Villarejo Ramírez, C. (1998): Análisis del discurso de la prensa sobre las encuestas electorales en las elecciones generales de 1996. Empiria, n. ${ }^{\circ}$ 1. UNED. Madrid.

ArmatTe, M. (1995): Histoire du modèle linéaire. Formes et usages en statistique et econometrie jusqu'en 1945 (Thèse de Doctorat). Paris, EHESS.

- (2003): Phierre Thionet et l' inroduction en France des méthodes de sondage aléatoire. Journal de la Société Française de Statistique, tome 44, n. ${ }^{\circ}$ 1-2.

ARRIBAS MACHO, J. M. (1998): El modelo estadístico desde la perspectiva cualitativa. Empiria, n. ${ }^{\circ}$ 1. UNED. Madrid.

- (2004): «Los comienzos de la estadística matemática (1914-1936)». Historia de la Probabilidad y la Estadística (II). AHEPE. Madrid, Delta publicaciones.

BEAUD, P. et QUERE, L. (1990): La formation de l'opinion comme phénomène intersubjectif. Pour un changement de paradigme dans l'étude de l'opinion publique. Rapport de recherche pour la Convention C.N.E.T. / M.S.H «Étude des mécanismes de formation et de production des opinions». Paris.

Bernouilli, N. (1709): De Usu Artis Conjectandi in Jure (Thèse doctorant, Bâle, 1709) Traducción de Norbert Meusnier (1991) Paris: EHESS/CAMS.

BLoNDIAUX, L. (1998): La fabrique de l'opinion. Une histoire sociale des sondages. Paris, Seuil.

BOREL, E. (1908a): Le calcul des probabilités et la méthode des majorités. Année psychologique, t. 14, pp. 125-151.

- (1908b): Le calcul des probabilités et la mentalité individualiste. Revue de Mois, t. 6, pp. 641-650.

BouzA, F.: Desventuras De un concepto claro y distinto: Opinión Pública, en http://www.ucm.es//info//socvi/BOUZA/NUEVA 1/Textos/

Camarero, L. (2001): Los soportes de la encuesta: la infancia de los métodos representativos. Metodología de Encuestas, vol. 3, n. ${ }^{\circ} 2$.

Champagne, P. (1990): Faire l'opinion. Le nouveau jeu politique. Paris, Les Éditions de Minuit.

CRAMER, G. (1740, fecha aproximada de aparición): «Probabilité des témoignages». Cours de logique.

DE MORA, M. S. (2004): «El problema del testimonio». Historia de la Probabilidad y la Estadística (II). Madrid: AHEPE. Delta publicaciones.

DESROSIÈRES, A. (2004): La política de los grandes números. Barcelona, Melusina.

DIDEROT (1765): L'Encyclopédie (Dictionnaire raisonné des sciences, des arts et des métiers). Paris: Edición de 1765, pp. 393-400.

DONELLY, M. (1996): «From political arithmetic to social statistics: how some nineteenthcentury roots of the social sciences were implanted». The rise of the social sciences and the formation of modernity. Conceptual change in context, 1750-1850. London, Kluwer Academic Publishers.

FARGE, A. (1992): Dire et mal dire. L'opinion publique au XVIIIe siècle . Paris, Seuil.

GrofmAN, B. et FELD, S. L. (1989): La volonté générale de Rousseau: une perspective condorcetienne. Condorcet, mathématicien, économistte, philosophe, homme politique. Colloque international. Minerve. 
Gumbel, E. J. (1928): Statistique et lutte des classes. Réflexion programmatique (Traducción del alemán por Hertz, S.) Mathématiques et Action politique. Études d'histoire et de philosophie des mathématiques sociales. Institut National d'Études Démographiques. Paris. 2000.

HABermas, J. (1994): Historia y crítica de la opinión pública. Barcelona: Ediciones G. Gilli, S. A. (4. ${ }^{\mathrm{a}}$ ed.).

HERTZ, S. (2000): Statistique de l'État et statistique mathématique. Un texte-manifeste remarquable d'Emil julius Gumbel: "Statistique et lutte des classes» (1928). Mathématiques et Action politique. Études d'histoire et de philosophie des mathématiques sociales. Institut National d'Études Démographiques. Paris.

MARTIn, O. (1997): La mesure de l'esprit. Origines et développements de la psychométrie, 1900-1950. Paris, Éditions L'Harmattan.

- (1999): Raison statistique et raison sociologique chez Maurice Halbwachs. Maurice Halbwachs et les sciences humaines de son temps. Revue d'Histoire des Sciences Humaines, 1. Presses universitaires Septentrion., pp. 69-101.

MARTIN, O. et VANNIER, P. (2002): La sociologie française après 1945: places et rôles des méthodes issues de la psychologie. Mathématiques et sciences sociales au cours du $\mathrm{XX}^{\mathrm{e}}$ siècle. Revue d'Histoire de Sciences Humaines, $n{ }^{\circ} 6$. Presses universitaires du Septemtrion., pp. 108-113

MEUSNIER, N. (1995): La passe de l'esperance. L'emergence d' une mathématique du probable au XVII siècle. Mathématiques informatique et sciences humaines (33e année, n. $\left.{ }^{\circ} 131\right)$, pp. 5-28.

Pertué, M. (1989): La censure du peuple dans le projet de Constitution de Condorcet. Condorcet, mathématicien, économiste, philosophe, homme politique. Colloque international. Minerve.

PORTER, T. M. (1995): Trust in Numbers. The pursuit of objectivity in science and public life. Princeton University Press.

RifFAULT, H. (1981): L'institut français d'opinion publique 1938-1978. Science et théorie de l'opinion publique, Hommage à Jean Stoetzel. Paris: RETZ.

SCHWEBER, L. (2002): Wartime research and the quantification of American sociology. The view from «The American Soldier». Mathématiques et sciences sociales au cours du XX ${ }^{\mathrm{e}}$ siècle. Revue d'Histoire de Sciences Humaines, n. $^{\circ}$ 6. Presses universitaires du Septemtrion.

Stoetzel, J. et GiRARD, A. (1973): Les sondages d'opinion. Paris, Presses Universitaires de France.

TASSI, P. (1988): «De l'exhaustif au partiel: un peu d'histoire sur le développement des sondages». Estimation et Sondages. Cinq conributions à l' histoire de la statistique. Paris, Economica. 


\section{RESUMEN}

El presente artículo pretende realizar un recorrido por los avatares que ha ido experimentando el concepto de opinión pública, desde que aparece como tal a mediados del siglo XVIII hasta el momento del triunfo definitivo de la metodología que, hasta el día de hoy, ha sido predominante como técnica de investigación social en las democracias occidentales a partir de los años treinta del siglo Xx: la encuesta estadística. Así, las perspectivas teórica y metodológica se relacionan y complementan, ya que el propio concepto de opinión pública se verá influido y mediatizado por la propia metodología que en cada momento ha sido utilizada para «registrarlo», «medirlo», en definitiva, para dar cuenta del mismo.

Partiendo de este planteamiento, trataremos de presentar, no sólo los comienzos de la noción de opinión pública, sino también - y fundamentalmentelos orígenes matemáticos de los sondeos, a través de las representaciones que, sobre aquellas entidades que no resultan tan fácil ni evidentemente medibles como las del «mundo físico» — tales como las opiniones —, van a mostrar los autores de la temprana estadística social, obviamente influidos por el contexto político y social de su época.

Finalmente realizaremos un breve repaso por el proceso mediante el cual los sondeos de opinión «arrancan» en el contexto norteamericano de los años treinta y terminan por adquirir la «legitimación necesaria» para convertirse en la metodología predominante en la investigación de la opinión pública.

\section{PALABAS CLAVE}

Historia de la estadística, encuesta, opinión pública.

\section{ABSTRACT}

This article tries to follow the changes involved in the development of the concept «public opinion» since its first use in the middle of the 18th century until statistical surveying became predominant in social research in western democracies from the 1930s. It is argued that theoretical and methodological perspectives are not only related but supplementary, and that the concept of «public opinion" is influenced and mediatized by the methodology which is being used to «register», to «measure».

The paper outlines not only the beginnings of the notion of public opinion, but also the mathematical origins of polls through the representations of entities which are not easily appraisable or evidently part of the «physical world» - such as opinions - by early authors of social statistics, obviously influenced by the political and social context of their time. 
The paper concludes with a brief consideration of the rise of opinion polls in the U.S. in the 1930s, acquiring the «necessary legitimation» to become the predominant methodology in public opinion investigation.

\section{KEY WORDS}

History of stadistics, survey, public opinion. 